\title{
A Case of ‘Epilepsy’ Successfully Treated by Cardiac Pacing
}

\author{
Mai W McCrea
}

$M B, B C h, M R C P(U K), R A M C^{*}$

Senior Registrar

DrLJ Findley

TD, MD, FRCP

Consultant Neurologist

Department of Medicine, Queen Elizabeth Military Hospital, Woolwich, London SE184QH

SUMMARY: We describe the case of a 41 year old male whose symptoms of episodes of disturbance of consciousness failed to respond to a trial of anticonvulsant therapy but, subsequent to demonstration of carotid sinus hypersensitivity, were successfully treated by permanent cardiac pacing.

\section{Introduction}

Carotid sinus hypersensitivity is a recognised cause of dizziness, syncope and convulsions (1-5). Unless it is suspected, patients may be diagnosed as suffering from epilepsy $(1,3,4,5)$.

Carotid sinus hypersensitivity has been defined as either ventricular asystole for more than three seconds (cardioinhibitory type) $(4,6,7,8)$ and/or substantial decrease $(30-50 \mathrm{mmHg}$, or greater) in systolic blood pressure (vaso-depressor type) (4) in response to continuous manual carotid sinus stimulation for a period of up to thirty seconds. Therefore, the diagnosis of the cardioinhibitory type of carotid sinus hypersensitivity requires that the patient is subjected to carotid sinus massage with simultaneous recording and observation of an electrocardiogram (ECG).

We describe a 41 year old soldier with a history of episodic disturbance of consciousness who had undergone extensive cardiological investigations and who was afterwards referred for neurological assessment with a provisional diagnosis of epilepsy.

Subsequent to neurological referral, he was found to have a hypersensitive carotid sinus reflex.

\section{Case Report}

The patient, a right handed male, was referred for advice concerning anti-convulsant therapy. Five years previously, he had been admitted to hospital following recurrent stereotyped episodes of dizziness, transient loss of consciousness and paraesthesiae of the right upper limb. Clinical examination revealed minimal weakness of the right arm. The neurological deficit resolved within 12 hours and aspirin therapy had been initiated. The soldier continued to experience these stereotyped episodes at a frequency of approximately once every 28 days.

Investigations including magnetic resonance imaging of the brain, digital subtraction carotid angiography and two dimensional echocardiography were normal. Six 24 hour Holter ambulatory ECG's demonstrated sinus rhythm with heart rate varying between 36 to 120 (mode *now H/SRoyal Army Medical College, Millbank, London SWIP4RJ
50) per minute. Unfortunately, the patient had been asymptomatic during these episodes of Holter ECG monitoring.

On one occasion, a medical officer witnessed the soldier to suddenly become unresponsive, with upward deviation of the eyes lasting approximately 90 seconds, after which the patient regained consciousness but showed paresis of the right arm. During this witnessed episode the patient's pulse was noted to be strong andto regular at a rate of 40 per minute. There was no history of urinary incontinence, tongue biting or jerking movements of the limbs in association with these events.

Although several stress (i.e. performed under conditions of fasting and sleep deprivation) and 24 hour ambulatory electroencephalograms had failed to demonstrate any abnormal findings, on account of the clinical picture, a diagnosis of cerebral dysrhythmia was made and carbamazepine was prescribed. The soldier was medically downgraded to P7 HO Ex NI and was instructed not to drive a motor vehicle, not to handle live ammunition and weapons and to avoid indulging in dangerous (e.g. working at heights) tasks. In view of his military experience, the patient was permitted to complete the remaining three years of his Army service. However, during this period the symptoms continued despite adequate plasma levels of carbamazepine. In addition, the soldier complained of headaches sometimes preceding his episodes of disturbance of consciousness and, in view of the possibility of complicated migraine, pizoytifen and mianserin hydrochloride were added to his medication. However, this therapeutic manoeuvre did not produce any tangible benefit for the patient.

Subsequent to leaving the Army, the patient continued to experience his symptoms and his civilian general practitioner unsuccessfully attempted to remedy the situation by adding phenytoin to the anti-convulsant regime. The patient was then referred for neurological advice concerning anti-epileptic therapy.

At this neurological referral, there were no abnormal clinical findings apart from bradycardia. There was no 


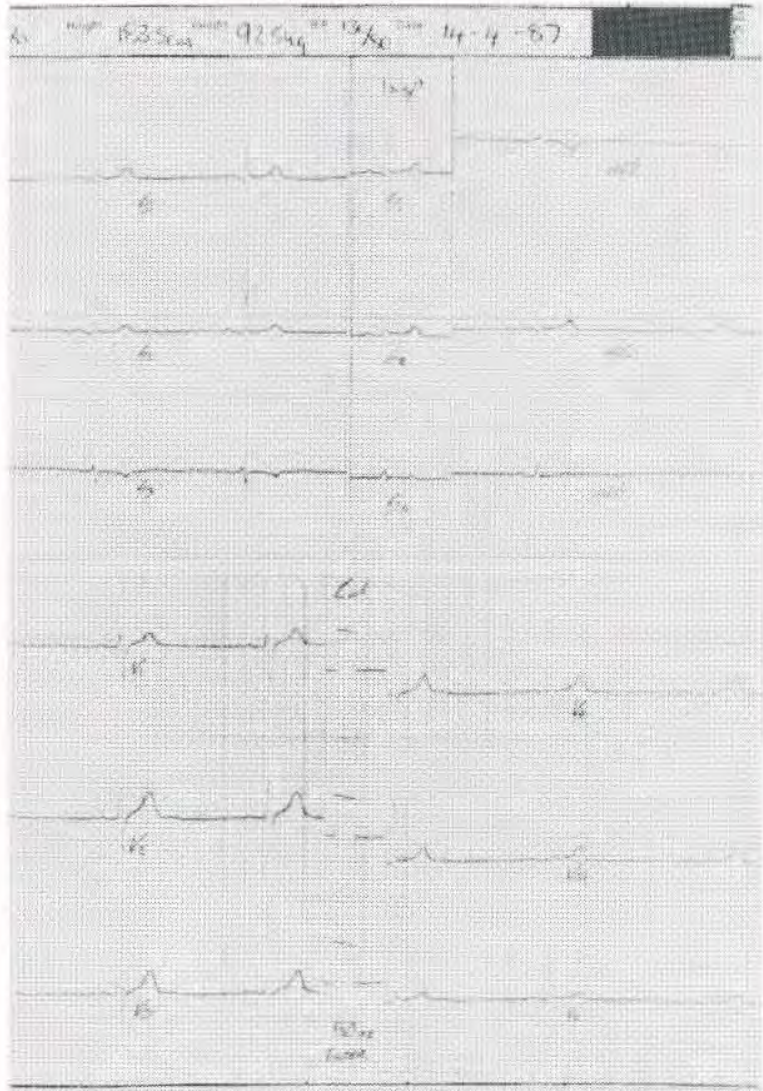

D:stow

Fig 1. Patient's resting twelve lead ECG demonstrating sinus bradycardia (heart rate 39 per minute) with normal axis and no evidence of conduction defect.

evidence of postural hypotension. ECG confirmed sinus rhythm (rate 39 per minute) with normal axis and no evidence of conduction defect (Fig 1). Carotid sinus massage to the right carotid artery within 2.8 seconds produced ventricular asystole of 4.4 seconds duration (Fig 2) associated with symptoms of dizziness. On recovery of cardiac rhythm, the patient complained of paraesthesiae in the right upper limb.

The patient was subsequently referred for cardiological opinion on the need for permanent cardiac pacing. A dual chamber pacing system was inserted and all of the patient's medication was discontinued. The patient has been completely asymptomatic in the twelve months since his pacemaker was inserted and his anticonvulsant medication was withdrawn. In addition, the patient has successfully applied for restoration of his driving licence.

\section{Discussion}

Prior to detection of his carotid sinus hypersensitivity this patient had been subjected to six periods of twenty-

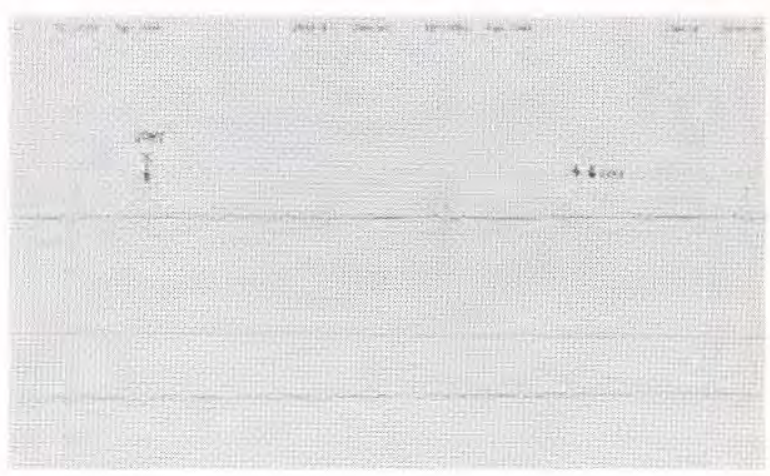

Fig 2. ECG demonstrating ventricular asystole of 4.4 seconds occurring after 2.8 seconds of massage to the patient's right carotid sinus.

four hour Holter ECG monitoring. These recordings had repeatedly failed to detect evidence of either spontaneous sinus node arrest or atrio-ventricular nodal block. However, absence of these abnormalities on ECG monitoring does not exclude carotid sinus syncope (2).

It is generally recognised that after carotid sinus massage of up to 30 seconds, the cardio-inhibitory type of carotid sinus hypersensitivity can be demonstrated in 25 per cent of asymptomatic individuals (9) and in alo least $40 \%$ of patients with cardiovascular disease (10) However, when carotid sinus stimulation is limited to five seconds and performed under standardised condi tions, hypersensitivity is elicited in only two per cent of the normal population (1). Thus, is should be eme phasised that carotid sinus syndrome is often a pre sumptive diagnosis (4) but the reproduction of the? patient's symptoms on carotid sinus massage, in association with ECG evidence of ventricular asystole, is highly supportive $(1,4)$.

Our patient persistently exhibited a resting ECG with bradycardia in the absence of any medication known to affect heart rate. This is suggestive of increased vagal tone which is associated with hypersensitivity of the carotid sinus $(1,8)$.

In conclusion, we suggest that patients with unexplained episodic disturbance of consciousness should be subjected to carotid sinus massage under controlled conditions (ECG monitoring). Otherwise, a diagnosis of carotid sinus hypersensitivity will be missed.

\section{REFERENCES}

1. Davies A B, Stephens M R, Davies A G. Carotid sinus hypersensitivity in patients presenting with syncope. Br Heart J 1979; 42: 583-586.

2. Walter P F, Crawley i S, Dorney E R. Carotid sinus hypersensitivity and syncope. Am J Cardiol 1978; 42: 396-403.

3. WeISS S, BAKER J. The carotid sinus reflex in health and disease. Its role in the causation of fainting and convulsions. Medicine (Baltimore) 1933; 12: 297 354. 
4. Thomas J E. Hyperactive carotid sinus reflex and carotid sinus syncope. Mayo Clinic Proc 1969; 127. 139.

5. Cohen F L, Fruehan C T, King R. Carotid sinus syndrome. J Neurosurg 1976; 45: 78-84.

6. Report of joint American College of Cardiology/ American Heart Association. Guidelines for permanent pacemaker implantation. Circulation 1984; 70: 331A-339A.

7. Recommendations for pacemaker prescription for symptomatic bradycardia. Report of a working party of the British Pacing and Electrophysiology Group. Br Heart J 1991; 66: 185-191.

8. Thormann J, Schwarz F. Vagal role and pacemaker indication in hypersensitive carotid sinus reflex. Eur J Cardiol 1975; 3: 47-51.

9. Heidorn G H, MeNamara A P. Effect of carotid sinus stimulation on the electrocardiograms of clinically normal individuals. Circulation 1956; 14: 1104-1113.

10. Sigler L H. The cardio-inhibitory carotid sinus reflex. Am J Cardiol 1963; 12: 174-183. 